\title{
MESSENGER Observations of Extreme Loading and Unloading of Mercury's Magnetic Tail
}

James A. Slavin ${ }^{1 *}$, Brian J. Anderson ${ }^{2}$, Daniel N. Baker ${ }^{3}$, Mehdi Benna ${ }^{4,5}$, Scott A. Boardsen $^{1,5}$, George Gloeckler ${ }^{6,7}$, Robert E. Gold ${ }^{2}$, George C. Ho ${ }^{2}$, Haje Korth ${ }^{2}$, Stamatios M. Krimigis ${ }^{2,8}$, Ralph L. McNutt, Jr. ${ }^{2}$, Larry R. Nittler ${ }^{9}$, Jim M. Raines $^{6}$, Menelaos Sarantos ${ }^{1,5}$, David Schriver ${ }^{10}$, Sean C. Solomon ${ }^{9}$, Richard D. Starr ${ }^{11}$, Pavel M. Trávníček ${ }^{10,12}$, Thomas H. Zurbuchen ${ }^{6}$

${ }^{1}$ Heliophysics Science Division, NASA Goddard Space Flight Center, Greenbelt, MD 20771, USA; ${ }^{2}$ The Johns Hopkins University Applied Physics Laboratory, Laurel, MD 20723, USA; ${ }^{3}$ Laboratory for Atmospheric and Space Physics, University of Colorado Boulder, CO 80303, USA; ${ }^{4}$ Solar System Exploration Division, NASA Goddard Space Flight Center, Greenbelt, MD 20771, USA; ${ }^{5}$ Goddard Earth Science and Technology Center, University of Maryland, Baltimore County, Baltimore, MD 21228, USA; ${ }^{6}$ Department of Astronomy, University of Maryland, College Park, MD 20742, USA; ${ }^{7}$ Department of Atmospheric, Oceanic and Space Sciences, The University of Michigan, Ann Arbor, MI 48109, USA; ${ }^{8}$ Academy of Athens, Athens 11527, Greece; ${ }^{9}$ Department of Terrestrial Magnetism, Carnegie Institution of Washington, Washington, DC 20015, USA; ${ }^{10}$ Institute of Geophysics and Planetary Physics, University of California, Los Angeles, CA 90024, USA; ${ }^{11}$ Department of Physics, Catholic University of America, Washington, DC 20064, USA; ${ }^{12}$ Astronomical Institute, Academy of Sciences of the Czech Republic, Prague, Czech Republic, 14131 .

*Corresponding author contact information: james.a.slavin@nasa.gov; phone 301-286-5839; fax: 301-286-5348.

During MESSENGER's third flyby of Mercury, a series of 2-3 minute long enhancements of the magnetic field in the planet's magnetotail were observed. Magnetospheric substorms at Earth are powered by similar tail loading, but the amplitude is $\sim 10$ times less and the durations are $\sim 1 \mathrm{hr}$. These observations of extreme loading imply that the relative intensity of substorms at Mercury must be much larger than at Earth. The correspondence between the duration of tail enhancements and the calculated $\sim 2$ min Dungey cycle, which describes plasma circulation through Mercury's magnetosphere, suggests that such circulation determines substorm timescale. A key aspect of tail unloading during terrestrial substorms is the acceleration of energetic charged particles. Such signatures are puzzlingly absent from the MESSENGER flyby measurements.

Magnetospheric substorms are space-weather disturbances powered by the rapid release of magnetic energy stored in the lobes of planetary magnetic tails (1). This loading and unloading of the Earth's tail occurs on timescales of $\sim 1 \mathrm{hr}$ and is closely 
correlated with a southward interplanetary magnetic field (IMF) (i.e., opposite to the planetary magnetic field at the nose of the magnetosphere), a geometry that drives magnetic flux loading into the tail via magnetic reconnection between the IMF and the dayside geomagnetic field (2). During a substorm, the accumulated magnetic energy is unloaded through reconnection of the oppositely directed magnetic fields in the tail lobes, resulting in the ejection of plasmoids, high-speed sunward and anti-sunward jetting of hot plasma, acceleration and injection of charged particles into the inner magnetosphere, and field-aligned currents flowing between the tail and the highlatitude atmosphere where aurorae are produced (3).

This circulation of plasma, magnetic flux, and energy from the dayside $\mathrm{X}$-line at the magnetopause to the nightside $\mathrm{X}$-line in the cross-tail current layer and, later, back to the dayside magnetosphere constitutes the "Dungey cycle" (4) whose energy is drawn from the solar wind. The large magnetic field normal to the magnetopause measured during the second MESSENGER flyby when the IMF was southward was used to calculate a cross-magnetosphere electric potential of $\sim 30 \mathrm{kV}$ or a mean dawnto-dusk electric field of $\sim 2 \mathrm{mV} / \mathrm{m}$ (5). This electric field implies a Dungey cycle time (i.e., time to $E \times B$ drift from local noon to midnight in the polar cap or from the northern boundary of the tail down to the cross-tail current sheet) at Mercury of $\sim 2$ $\min$. The $\sim 1 \mathrm{hr}$ Dungey cycle time at Earth is believed to be the underlying reason for the $\sim 1-3 \mathrm{hr}$ duration of terrestrial substorms $(1,4)$.

The IMF immediatedly preceding MESSENGER's third flyby had a variable northsouth orientation and had a magnitude of $\sim 28 \mathrm{nT}, \sim 50 \%$ stronger than all other encounters. Like the other MESSENGER flybys, the M3 trajectory was near equatorial with the spacecraft entering the magnetosphere through the downstream dusk magnetosheath and exiting just forward of the dawn terminator (Fig. 1). The inbound bow shock (BS) and (average) magnetopause (MP) crossing times were 20:56:06 and 21:27:45 UTC, respectively. The MESSENGER spacecraft autonomously terminated science observations and entered a "safe-hold" at 21:48:37 UTC, so no outbound boundary crossings were measured. A fit to the MESSENGER and Mariner 10 averaged boundary crossings using methods and functional forms recently applied to 
Mercury (6-8) yield mean subsolar bow shock and magnetopause planetocentric distances of 1.7 and $1.3 R_{\mathrm{M}}$, respectively, where $R_{\mathrm{M}}$ is Mercury's radius (Figs. 1).

Following the magnetopause crossing, magnetometer data (10) were acquired spanning the dusk-side tail as the spacecraft moved from $X_{\mathrm{MSO}}=-1.85$ to $-1.29 R_{\mathrm{M}}$ and $Y_{\mathrm{MSO}}=2.40$ to $0.16 R_{\mathrm{M}}$. Within the magnetosphere, the magnetic field data (Fig. 2) show a predominanlty strong negative $B_{\mathrm{X}}$ indicating that the spacecraft entered Mercury's magnetic tail through the southern lobe and remained there for about 20 min. There were several brief encounters with the plasma sheet, during which the field strength was temporarily depressed.

During the four intervals labeled events $1,2,3$ and 4, each lasting 2 to 3 minutes, the magnitude of the magnetic field in Mercury' tail increased and then decreased by factors of $\sim 2$ to 3.5 (Fig. 2). Events 2-4 corresponded to higher $\left|B_{Y} / B_{X}\right|$ than the intervening periods indicating increased magnetotail flaring. The magnetic field in the tail is in pressure equilibrium with the external solar wind. The tail magnetic field increases because of either enhanced external solar wind pressure or loading of the tail with additional magnetic flux. The latter process forces the magnetopause to flare outwards and increase the angle of incidence of the solar wind on the tail magnetopause. The $\left|B_{\mathrm{Y}} / B_{\mathrm{X}}\right|$ signatures of greater tail flaring imply that the field increases were due to flux loading of the tail (2).

Event 1 was observed at the outer edge of the tail, where more than a dozen transitions between the magnetosheath and magnetosphere occurred most likely due to large-amplitude Kelvin-Helmholtz boundary waves (12) as observed in similar regions at Earth (13). In addition to the boundary wave signatures, an overall increase in the tail magnetic field strength to $56 \mathrm{nT}$ followed by a decrease to $\sim 20 \mathrm{nT}$ is apparent (Fig. 2) and is labled Event 1. Event 2 was similar in duration, $\sim 2 \mathrm{~min}$, but larger in amplitude, with the magnetic field increasing from $\sim 20 \mathrm{nT}$ to $70 \mathrm{nT}$ before decreasing (Fig. 3). Events 3 and 4 (Fig. 2) were similar in duration and had peak magnetic field intensities of 83 and $70 \mathrm{nT}$, respectively.

Intense substorms in the terrestrial magnetosphere are associated with increases in tail magnetic field of $\sim 25 \%(14,15)$. Given that magnetic energy density is 
proportional to the square of magnitude, and neglecting changes in tail diameter, the increase in Earth's tail magnetic energy content during a loading event is less than a factor of $\sim 1.6$, whereas the present observations imply that Mercury's tail magnetic energy content increased by factors as great as $\sim 10$.

The amount of magnetic flux threading each tail, neglecting the small contribution from the plasma sheet, may be simply estimated as:

$$
\Phi_{\mathrm{TAIL}}=0.5 \pi B_{\mathrm{TAIL}} R_{\mathrm{TAIL}}^{2}
$$

where $B_{\text {TAIL }}$ is the field strengthin the lobe region and $R_{\text {TAIL }}$ is the radius of the tail. Event 1 occurred during multiple magnetopause crossings, indicating $R_{\mathrm{TAIL}} \sim 2.4 R_{\mathrm{M}}$ at $\mathrm{X}_{\mathrm{MSO}}=-1.8 \mathrm{R}_{\mathrm{M}}$. From the peak magnetic field, $56 \mathrm{nT}$, we computed a tail flux content of 3.0 MWb. For the other three events, the peak tail field intensities were 70, 83, and $70 \mathrm{nT}$, respectively. The increased radius of the tail accompanying these loading events was not measured by MESSENGER because the spacecraft was too deep in the tail to encounter the magnetopause. The pressure balances along the magnetopause (2), the peak loading field intensities, and the solar wind conditions predicted from a magnetohydrodynamic model of the inner heliosphere driven by solar magnetic field observations (16), imply a tail flaring angle relative to the sunward direction of $\sim 30^{\circ}$ for the strongest loading event, 3 , in contrast to $\sim 10^{\circ}$ for the much weaker event 1 . Such strong flaring implies a substantial enhancement of tail radius relative to the first loading event. Guided by these simulations and given the magnetospheric dimensions and the intensity of the inferred flaring, the radius of the tail for event 3 may have reached 3.5 $R_{\mathrm{M}}$ corresponding to a peak tail flux content of 9.5 MWb. This value is $\sim 50 \%$ more than predicted by a recently developed magnetospheric model of Mercury's magnetosphere (17) at the time of the second flyby, during which no loading events were observed.

Closer inspection of the magnetic field record for event 2 (Fig. 3) reveals six intervals of several seconds each when the total magnetic field weakened, indicating entry into a region with high plasma thermal pressure and low magnetic field pressure. These minima in field magnitude coincide with either rapid northward-then-southward or just southward variations in $B_{Z}$, followed by a slower recovery back to $B_{Z} \sim 0$, as can be seen in the latitude angle of the field (Fig. 3). These characteristics are 
signatures of plasmoids moving anti-sunward over the spacecraft $(18,19,20)$. The field near the peak of event 3 does not show marked intensity decreases but, a series of compressions is observed coincident with southward-then-northward tilting of the lobe magnetic field. These are signatures of traveling compression regions (TCRs) produced by the lobe magnetic field draping about sunward-moving flux ropes (21, 22). A transition from plasmoids being ejected tailward to sunward-moving TCRs closer to Mercury indicates the location of most intense tail reconnection $(1,2)$, the near-Mercury neutral line (NMNL). The NMNL was observed near $X_{\mathrm{MSO}}=-2.6 R_{\mathrm{M}}$ during MESSENGER's second flyby (5), but closer to the planet, near $X_{\mathrm{MSO}} \sim-1.6 R_{\mathrm{M}}$ for this third flyby. The third flyby results, therefore, suggest that the NMNL develops much closer to the planet when the magnetosphere is heavily loaded with magnetic flux, such as during events 2 and 3 .

The total magnetic flux emanating from Mercury's surface can be calculated for a simple centered dipole:

$$
\Phi_{\mathrm{M}}=2 \pi B_{\mathrm{eq}} R_{\mathrm{M}}{ }^{2}
$$

where $B_{\text {eq }}$ is the strengthof the magnetic field at Mercury's equator. Given $B_{\text {eq }} \sim 250$ $\mathrm{nT}(23,24)$, the corresponding value of $\Phi_{\mathrm{M}}$ is $9.5 \mathrm{MWb}$. As closed magnetic flux in the dayside magnetosphere is opened by reconnection at the magnetopause, it is pulled back into the tail lobes by the solar wind. For moderate loading of the tail, the dayside magnetopause contracts to lower altitudes and the north and south magnetic cusps are displaced equatorward (Fig. 4b). In the asymptotic limit that $100 \%$ of the planet's magnetic flux is transferred to the tail, the closed dayside magnetosphere disappears, the magnetopause flares strongly, and the north and south cusps merge into a single broader cusp at the equator (Fig. 4c). The tail flux contents at the time of the peak loading events measured by MESSENGER correspond to at least $\sim 30 \%$, and for the most intense event possibly $100 \%$, of the available magnetic flux from Mercury. Such an extreme magnetospheric configuration has never been observed or inferred to be present on the basis of space measurements at Earth or at other planets. The typical fraction of Earth's total magnetic flux that is contained in the tail during loading events producing intense substorms is only $\sim 10-12 \%$ (14). If Mercury's dayside magnetosphere is entirely depleted by reconnection, which may have occurred during 
event 3, the entire dayside surface would map to open magnetic field lines and be exposed to the shocked solar wind of the magnetosheath.

The close correspondence between the 2-3 min duration of the tail loading/unloading observed during the third flyby and the $\sim 2$ min Dungey cycle time suggests not only that Earth-like substorms occur at Mercury, but also that plasma circulation times determine the temporal scale for substorms at both planets. Further, relative loading and unloading variation in tail energy content observed by MESSENGER at Mercury was an order of magnitude larger than at Earth implying that the relative energy release in substorms at Mercury must be large compared to terrestrial substorms. The high rate of reconnection inferred from the large magnetopause-normal magnetic fields seen during MESSENGER's second flyby (5) and the large the flux transfer events observed just outside Mercury's magnetopause (25) by MESSENGER during its earlier flybys (5) are the most likely cause of this intense tail loading. For example, 10 of the largest flux transfer events measured during the second flyby over a period of $\sim 1-2 \mathrm{~min}$, or 1 FTE every 6 to $12 \mathrm{~s}$, would contribute $\sim 2 \mathrm{MWb}$ to the tail loading and account for a significant portion of the MESSENGER events. The intense fluxes of higher energy electrons reported by Mariner 10 and attributed to substorm behavior $(26,27)$, and the observations strong tail loading/unloading and plasmoid ejection reported here, make the lack of energetic particles with energies above $36 \mathrm{keV}$ in the MESSENGER observations (28) is even more surprising. The production of energetic particle acceleration events at Mercury, such as that observed by Mariner 10, evidently requires conditions not yet encountered by MESSENGER.

\section{References and Notes}

1. D. N. Baker, T. I. Pulkkinen, V. Angelopoulos, W. Baumjohann, R. L. McPherron, J. Geophys. Res. 101, 12975 (1996).

2. C. T. Russell, R. L. McPherron, Space Sci. Rev. 15, 205 (1973).

3. V. Angelopoulos et al., Science 321, 931 (2008).

4. G. L. Siscoe, N. F. Ness, C. M. Yeates, J. Geophys. Res. 80, 4359 (1975).

5. J. A. Slavin et al., Science 324, 606 (2009).

6. J.-H. Shue, J. K. Chao, H. C. Fu, C. T. Russell, P. Song, K. K. Khurana, H. J. Singer, J. Geophys. Res. 102, 9497 (1997) 
7. Magnetopause crossings were fit to the model (6) with $\alpha=0.5$ and $R_{\mathrm{o}}=1.3 R_{\mathrm{M}}$, where $\alpha$ is the surface flaring parameter and $R_{\mathrm{o}}$ is the distance to the subsolar magnetopause.

8. J. A. Slavin et al., Geophys. Res. Lett. 36, L02101, 10.1029/2008GL036158 (2009)

9. Bow shock crossings were fit to the model ( 8 ) with $X_{\mathrm{o}}=0.475, \varepsilon=1.04$, and $L=$ $2.59 R_{\mathrm{M}}$, where $X_{\mathrm{o}}$ is the location of the hyperbola's focus along the aberrated $X_{\mathrm{MSO}}$ axis, $\varepsilon$ is the surface's eccentricity, and $L$ is the semi-latus rectum.

10. B. J. Anderson et al., Space Sci. Rev. 131, 417 (2007).

11. S. A. Boardsen, J. A. Slavin, B. J. Anderson, H. Korth, and S. C. Solomon, Eos Trans. AGU, 90(52), Fall Meet. Suppl., abstract P21A-1205 (2009)

12. Travnicek, P.M., P. Hellinger, D. Schriver, D. Hercik, B.J. Anderson, M. Sarantos, J.A. Slavin and T. Zurbuchen, Icarus, doi:10.1016/j.icarus.2010.01.008 (2010).

13. H. Hasegawa, M. Fujimoto, T.-D. Phan, H. Rème, A. Balogh, M. W. Dunlop, C. Hashimoto and R. TanDokoro, Nature 430, 755 (2004).

14. S. E. Milan et al., J. Geophys. Res. 109, A04220, 10.1029/2003JA010350 (2004).

15. C.-S. Huang, A. D. DeJong, X. Cai, J. Geophys. Res. 114, A07202, 10.1029/2009JA014232 (2009).

16. D. Odstrcil, D. N. Baker, B. J. Anderson, L. R. Mayer, J. A. Slavin, S. C. Solomon, Eos Trans. AGU, 90(52), Fall Meet. Suppl., abstract P24A-02 (2009).

17. I. I. Alexeev et al., Eos Trans. AGU,90(22), Jt. Assem. Suppl., Abstract P33B-04, abstr., (2009).

18. E. W. Hones, Jr. et al., Geophys. Res. Lett. 11, 5 (1984).

19. J. A. Slavin et al., J. Geophys. Res. 108, 1015, 10.1029/2002JA009557 (2003).

20. A. Kidder, R. M. Winglee, E. M. Harnett, J. Geophys. Res. 113, A09223, 10.1029/2008JA013038 (2008).

21. M. B. Moldwin, W. J. Hughes, J. Geophys. Res. 98, 81 (1993).

22. J. A. Slavin et al., J. Geophys. Res. 110, A06207, 10.1029/2004JA010878, (2005).

23. B. J. Anderson et al., Science 321, 82 (2008).

24. B. J. Anderson et al., Space Sci. Rev., DOI 10.1007/s11214-009-9544-3 (2010).

25. J. A. Slavin et al., Geophys. Res. Lett. 37, L02105, 10.1029/2009GL041485 (2010).

26. D. N. Baker, J. A. Simpson, J. H. Eraker, J. Geophys. Res. 91, 8742 (1986).

27. S. P. Christon, J. Feynman, J. A. Slavin, in Magnetotail Physics, A. T. Y. Lui, Ed. (Johns Hopkins University Press, Baltimore, 1987), pp. 393-400.

28. R. D. Starr et al., Eos Trans. AGU, 90(52), Fall Meet. Suppl., abstract P21A-1195 (2009).

29. We thank all those who contributed to the success of the MESSENGER flybys of Mercury. Data visualization and graphics support by J. Feggans, and M. Marosy are gratefully acknowledged. The MESSENGER project is supported by the NASA Discovery Program under contracts NAS5-97271 to The Johns Hopkins University Applied Physics Laboratory and NASW-00002 to the Carnegie Institution of Washington. 
Figure Legends

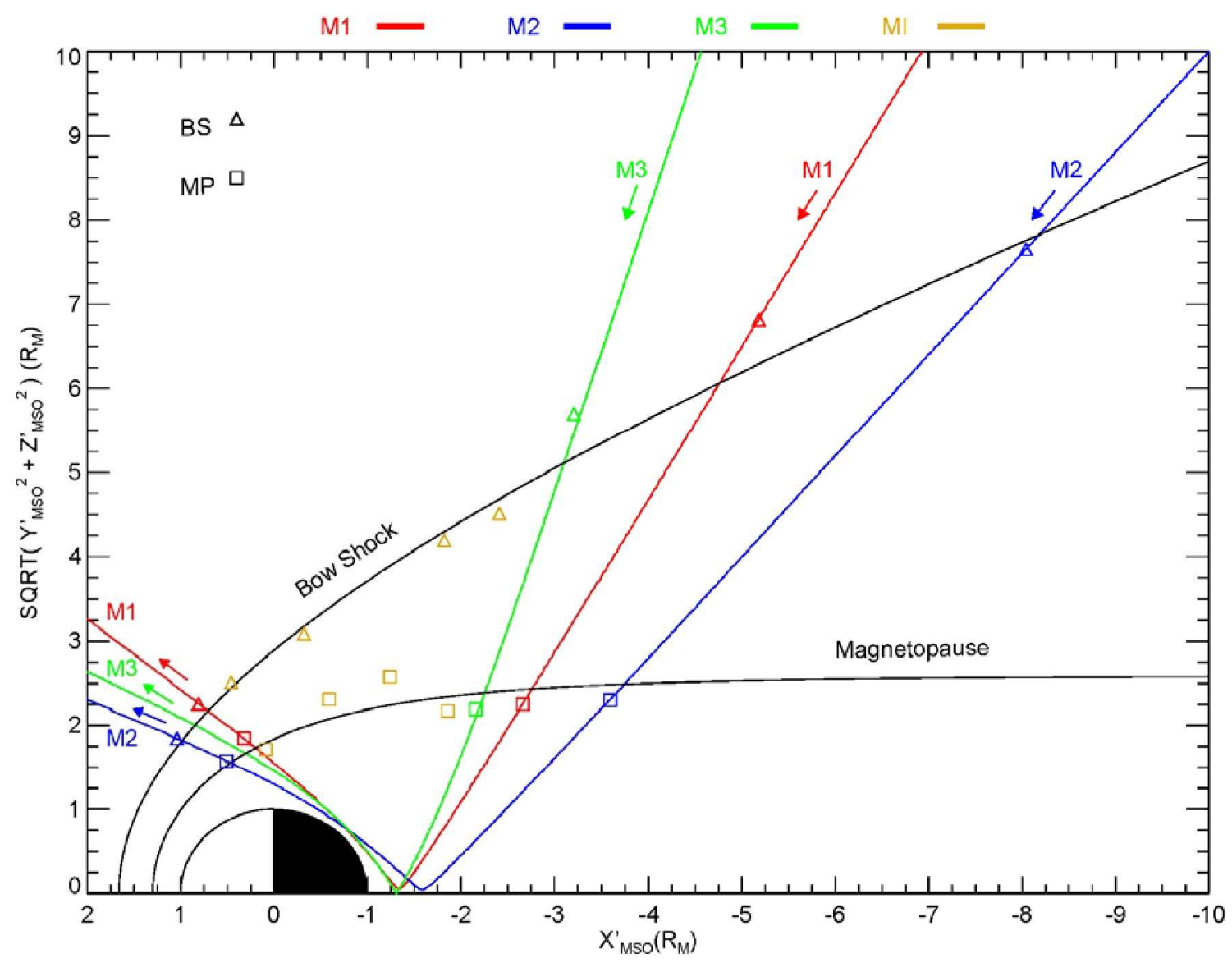

Fig. 1. MESSENGER Mercury flyby trajectories are displayed in solar-wind-aberrated cylindrical MSO coordinates. Averaged Mariner 10 and MESSENGER inbound and outbound bow shock (BS) and magnetopause (MP) crossings are shown as triangles and squares, respectively. Model boundary surfaces fit to all of the crossings are also displayed $(16,17)$. 


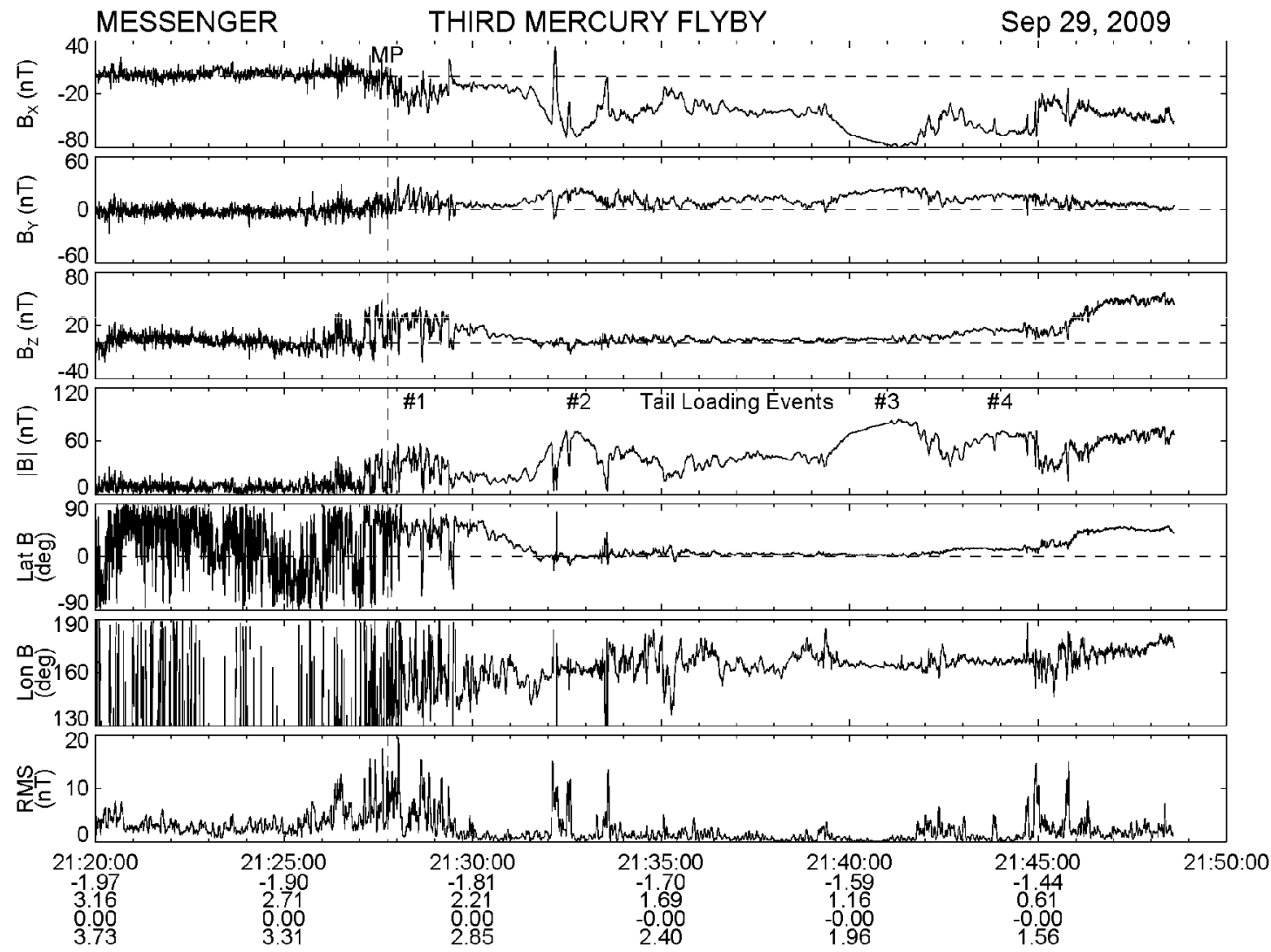

Fig. 2. Overview of magnetospheric measurements taken by MESSENGER's Magnetometer (MAG). The crossing from the magnetosheath into the magnetic tail is marked with a vertical dashed line. Closest approach (CA) was at an altitude of $228 \mathrm{~km}$ at 21:54:58 UTC. The MAG observations of magnetic field in MSO coordinates along with the latitude and longitude direction angles and the root-mean-squared (RMS) variance calculated over 3-s intervals are displayed in the top seven panels. In MSO coordinates $X_{\mathrm{MSO}}$ is directed from the center of the planet toward the Sun; $Z_{\mathrm{MSO}}$ is normal to Mercury's orbital plane and positive toward the north celestial pole; and $Y_{\text {MSo }}$ is positive in the direction opposite to orbital motion. The longitude angle of the magnetic field is defined to be $0^{\circ}$ toward the Sun and increases counter-clockwise looking down from the north celestial pole. The magnetic field latitude is $+90^{\circ}$ when directed northward and $0^{\circ}$ 
when it is in the $X_{\text {MSO }}-Y_{\text {MSo }}$ plane. The four tail loading events discussed here are labeled.

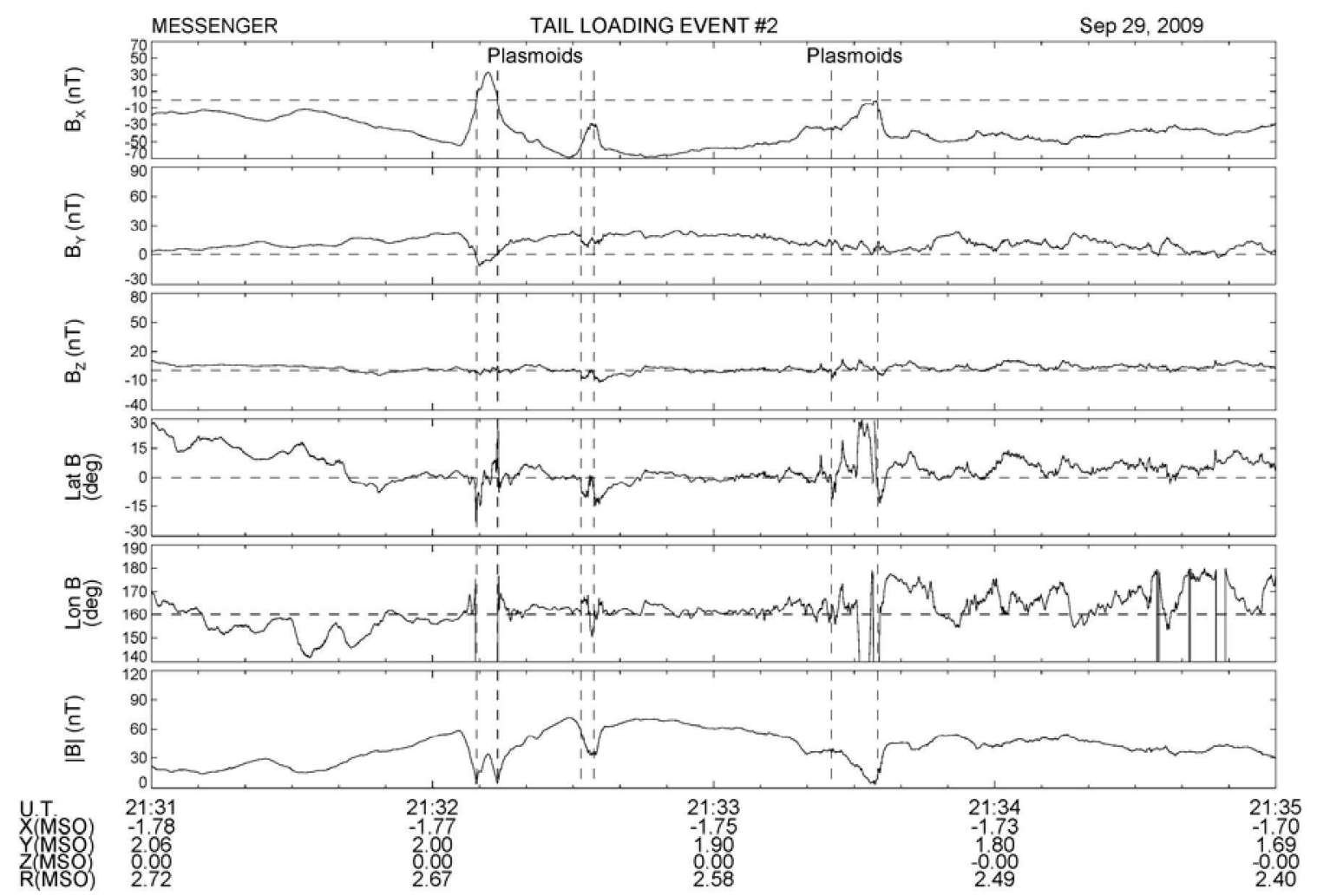

Fig. 3. Magnetometer observations of tail loading event 2 during MESSENGER's third flyby. Vertical dashed lines mark the occurrence of tailward-moving plasmoids. 


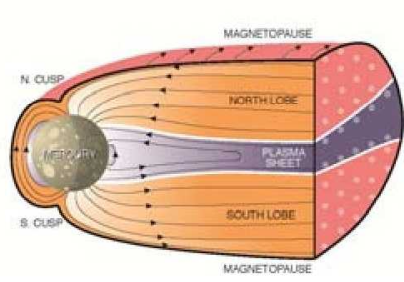

a. GROUnD STATE

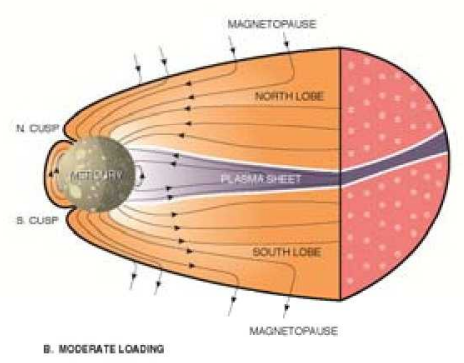

B. MESTRATE LOADHAOCOA

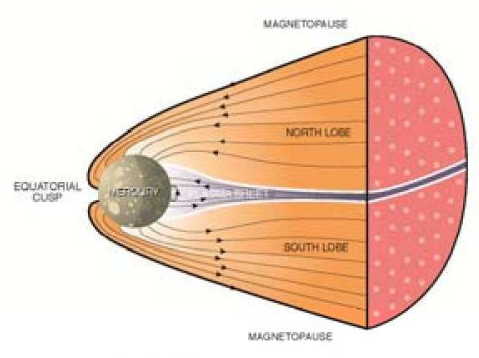

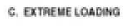

Fig. 4. Schematic view of Mercury's magnetosphere in its ground state and during moderate and extreme tail loading observed by MESSENGER on 29 September 2009. 